\title{
Produção e qualidade do melão em diferentes densidades de plantio
}

\author{
Geraldo M. de Resende; Nivaldo D. Costa
}

Embrapa Semi-Árido, C. Postal 23, 56300-000 Petrolina-PE; E-mail: gmilanez@ufla.br

\begin{abstract}
RESUMO
Avaliou-se a produção e qualidade do melão em diferentes densidades de plantio em experimento em Mandacaru, Juazeiro (BA), de setembro a novembro/98. O delineamento experimental foi de blocos ao acaso em parcelas subdivididas, sendo as parcelas constituídas pelos espaçamentos entre linhas $(1,80$ e $2,00 \mathrm{~m})$ e as subparcelas pelos espaçamentos entre plantas $(0,20 ; 0,40$ e $0,60 \mathrm{~m})$ correspondendo à densidades de plantio de 5,$00 ; 2,50$ e 1,66 plantas por metro linear de sulco, com quatro repetições. Utilizou-se a cultivar AF-682. A produção comercial diminuiu linearmente com a redução da densidade de plantio. O espaçamento de $0,20 \mathrm{~m}$ com 5,00 plantas metro/linear alcançou a maior produtividade com 40,69 $\mathrm{t} / \mathrm{ha}$. A massa fresca do fruto apresentou efeito linear positivo com a redução da densidade de plantio. $\mathrm{O}$ espaçamento de $0,60 \mathrm{~m}$ com 1,66 plantas por metro linear apresentou a maior massa fresca do fruto com 2,09 kg/fruto. Com o aumento do espaçamento de plantio registrou-se maior número de frutos por planta. A maioria dos frutos concentrou-se nos tipos 6 e 8, que são os de maior tamanho e que têm a preferência do mercado interno e em menor porcentagem para os tipos 10 e 12, que são os de melhor aceitação no mercado externo. A densidade de plantio não afetou os sólidos solúveis e a relação de formato do fruto.
\end{abstract}

Palavras-chave: Cucumis melo, rendimento, espaçamento, classificação de frutos, sólidos solúveis.

\begin{abstract}
Yield and fruit quality of melon in different planting densities

The yield and quality of melon AF 682 was evaluated in different planting densities in Juazeiro, Bahia State, Brazil. A randomized complete block design with split-plot and four replications was used, where main plots were made up of rows 1.8 and $2.0 \mathrm{~m}$ apart, and subplots were made up of three within-rows spacings $(0.2 ; 0.4$ and $0.6 \mathrm{~m}$ ), which corresponded to planting densities of $5.00 ; 2.50$ and 1.66 plants for linear furrow meter. The commercial yield decreased linearly with the planting density reduction. The stand of 5.00 plants per linear meter achieved the greatest yield $(40.69 \mathrm{t} / \mathrm{ha})$. The fresh mass of the fruits showed a linear positive effect with the planting density reduction. The stand of 1.66 plants per linear meter showed the greatest fruit fresh mass $(2.09 \mathrm{~kg} /$ fruit). Increasing the planting space, a greater number of fruits per plant was recorded. Most of the fruit was concentrated on the types 6 and 8, which was larger and had preference of the internal market. A smaller percentage was found for the types 10 and 12, which have better acceptance in the external market. Planting density did not affect the soluble solids content and the fruit shape ratio.
\end{abstract}

Keywords: Cucumis melo, spacing, fruits classification, soluble solids content.

\section{(Recebido para publicação em 12 de fevereiro de 2003 e aceito em 18 de setembro de 2003)}

$\mathrm{O}$ cultivo do melão nos últimos anos tem apresentado expansão na área cultivada, principalmente nas regiões de clima semi-árido, colocando o país na condição de exportador, pela excelente qualidade dos frutos e pelas condições climáticas que possibilitam a colheita na época de entressafra de outros países (Almeida, 1992). Segundo a FAO (2002), a produção mundial em 2001 atingiu 21,3 milhões de toneladas e uma produtividade média de $18,5 \mathrm{t} / \mathrm{ha}$, tendo o Brasil produzido $150.000 \mathrm{t}$ e uma produtividade de 12,5 t/ha. Em 2000, a região Nordeste, representada pelos estados do Rio Grande do Norte, Ceará, Bahia e Pernambuco, respondeu por 93,4\% da produção do país (IBGE, 2002), não só para o abastecimento interno, mas principalmente, para a exportação (Makishima, 1991).

A região do Submédio São Francisco tem-se destacado no contexto nacional como a grande produtora dessa olerícola. Entretanto, sua produtividade de 15,0 t/ha, é considerada baixa. Um dos fatores apontados para explicar esta baixa produtividade é a densidade de plantio. É utilizada na região uma população de 10.000 plantas/ha, no espaçamento de 2,00 m entre linhas e $0,50 \mathrm{~m}$ entre plantas, enquanto no Rio Grande do Norte é empregada a densidade de 20.000 plantas/ha (Dias et $a l ., 1998)$. Os espaçamentos mais comuns adotados pelos produtores são 1,80 ou $2,00 \mathrm{~m}$ entre as fileiras e 0,50 até $1,50 \mathrm{~m}$ entre plantas, geralmente, com uma ou duas plantas por cova (Faria et al., 2000). As empresas com alto nível tecnológico têm adotado espaçamentos de 2,00 a 3,00 m entre linhas e 0,30 a $0,80 \mathrm{~m}$ entre plantas, com densidades variando de 4.167 a 16.667 plantas/ha. A densidade mais utilizada pelas grandes empresas é de 30.000 plantas/ha, com espaçamento de $2,00 \mathrm{~m}$ entre linhas e $0,50 \mathrm{~m}$ entre plantas, pro- porcionando uma produtividade de 61,75 t/ha (Almeida, 1992; Grangeiro, 1997; Grangeiro et al., 1999b).

O espaçamento ideal da cultura depende da cultivar, do nível de tecnologia empregado pelo produtor e, principalmente, da exigência do mercado com relação ao tamanho dos frutos. Em pequenas áreas, usa-se o espaçamento de $2,00 \mathrm{~m}$ entre linhas e 0,30 a $0,50 \mathrm{~m}$ entre plantas, totalizando de 10.000 a 16.666 plantas/ha. Os produtores que cultivam áreas extensas, com alto nível de insumos modernos, têm adotado espaçamentos de 2,00 a 3,00 m entre linhas e de 0,12 a $0,50 \mathrm{~m}$ dentro das linhas, perfazendo 20.000 a 80.000 plantas/ha, deixando uma planta por cova (Pedrosa, 1994). Altas densidades produzem grande número de frutos por área, mas com tamanho, peso e número de frutos por planta reduzidos. Esse fato tem sido atribuído principalmente às pressões de competição inter e 
intraplantas (Robinson \& Walters, 1997). Nas baixas densidades tem sido verificado o inverso, ou seja, produção total menor com maior número de frutos por planta, de tamanho e peso mais elevados (Paris et al., 1988; Mendlinger, 1994).

O teor de açúcares dos frutos ( ${ }^{\circ}$ Brix $)$ está diretamente relacionado às condições climáticas durante a produção. Baixa umidade relativa do ar, aliada a temperaturas altas, favorece a produção de frutos com brix alto, que são os de melhor qualidade comercial e aqueles que alcançam boas cotações no mercado (Dusi, 1992).

O objetivo deste trabalho foi estabelecer a densidade de plantio mais adequada para o cultivo do melão cultivar AF-682 no Submédio São Francisco, visando maior produtividade e qualidade dos frutos.

\section{MATERIAL E MÉTODOS}

O experimento foi conduzido em campo da Embrapa Semi-Árido, em Juazeiro (BA), de setembro a novembro de 1998, em solo argiloso tipo Vertissolo com as características químicas e físicas na camada de $0-20 \mathrm{~cm}: \mathrm{K}=$ $0,35 \mathrm{cmol} / \mathrm{dm}^{-3} ; \mathrm{Ca}=30,1 \mathrm{cmol} / \mathrm{dm}^{-3}$ $\mathrm{Mg}=7,1 \mathrm{cmol}_{\mathrm{c}} / \mathrm{dm}^{-3} \mathrm{Na}=0,12 \mathrm{cmol}_{\mathrm{c}} /$ $\mathrm{dm}^{3} ; \mathrm{H}+\mathrm{Al}=0,0 \mathrm{cmol}_{\mathrm{c}} / \mathrm{dm}^{3} ; \mathrm{P}=29 \mathrm{mg}$ $\mathrm{dm}^{-3} ; 14 \mathrm{~g} \mathrm{~kg}^{-1}$ de M.Oे.; areia $=160 \mathrm{~g}$ $\mathrm{kg}^{-1}$; silte $=160 \mathrm{~g} \mathrm{~kg}^{-1} ;$ argila $=680 \mathrm{~g}$ $\mathrm{kg}^{-1}$ e 7,6 de $\mathrm{pH}$ em $\mathrm{H}_{2} 0$.

Adotou-se o delineamento experimental de blocos ao acaso, em parcelas subdivididas, com quatro repetições. As parcelas foram constituídas pelos espaçamentos entre linhas de 1,80 e 2,00 $\mathrm{m}$ e as subparcelas pelos espaçamentos entre plantas de 0,$20 ; 0,40$ e $0,60 \mathrm{~m}$, correspondendo a densidades de plantio de 5,$00 ; 2,50$ e 1,66 plantas por metro linear de sulco,e densidades variando de 8.300 a 27.000 plantas/ha. Foi utilizada a cultivar AF-682, de casca levemente enrugada, com coloração amarela ouro, cavidade interna pequena, uniforme, com massa fresca média entre 1,80 a $2,30 \mathrm{~kg}$ e brix médio de $12 \%$, apresentando tolerância/resistência ao vírus PRSV-W e à raça 1 e 2 de oídio (Alvarenga \& Resende, 2002).

A parcela experimental constituiuse de quatro linhas de 6,0 $\mathrm{m}$ de compri-

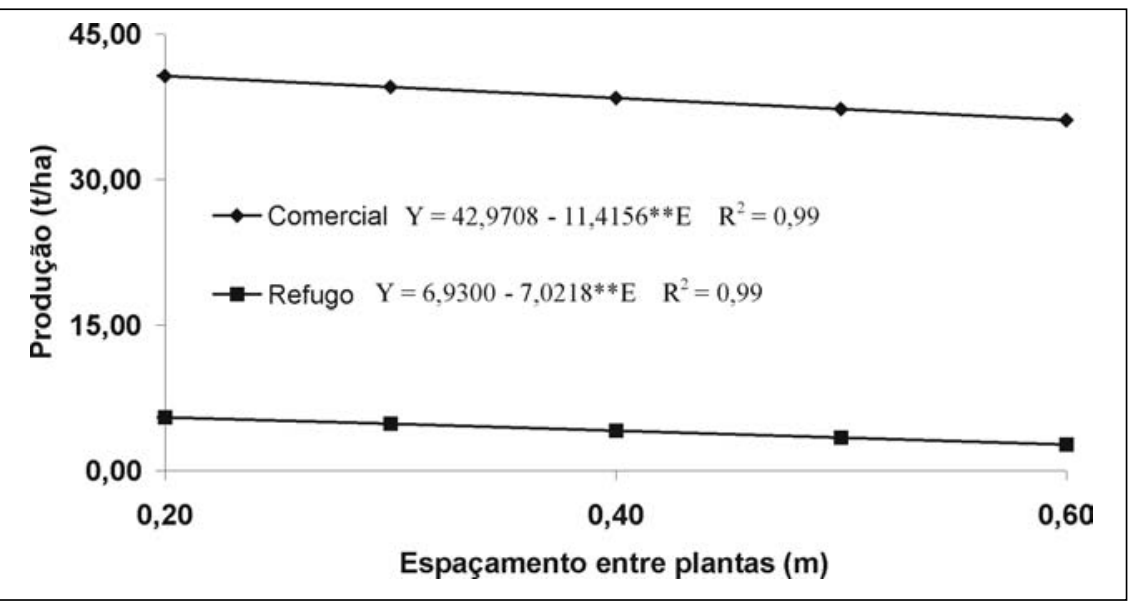

Figura 1. Produção comercial e refugo de melão em função do espaçamento entre plantas. Juazeiro(BA), Embrapa Semi-Árido, 1998.

mento, sendo considerada como área útil as duas linhas centrais. No plantio foram semeadas três sementes por cova, sendo quinze dias após realizado o desbaste, deixando-se uma planta por cova. A adubação de plantio, baseada na análise do solo, foi feita com $30 \mathrm{~kg} / \mathrm{ha}$ de $\mathrm{N}$, $120 \mathrm{~kg} / \mathrm{ha}$ de $\mathrm{P}_{2} \mathrm{O}_{5}$ e $60 \mathrm{~kg} / \mathrm{ha}$ de $\mathrm{K}_{2} \mathrm{O}$. A cobertura, em número de duas, foi realizadas com $30 \mathrm{~kg} /$ ha de $\mathrm{N}$ e de $\mathrm{K}_{2} \mathrm{O}$, aplicados aos 25 e 40 dias após a semeadura.

A irrigação foi feita por sulcos de infiltração em turno de rega de cinco dias, com lâminas em torno de $40 \mathrm{~mm}$, baseadas na evaporação da água medida no tanque classe A, sendo a cultura mantida no limpo através de capinas manuais. $\mathrm{O}$ raleio dos frutos foi feito retirando-se somente os frutos mal formados.

Adotou-se a coloração do fruto como critério para a colheita, quando os mesmos apresentavam-se totalmente amarelos. Foram realizadas duas colheitas, aos 65 e 75 dias após a semeadura. Obteve-se a produção de frutos comerciais [tipos 6 a 12 frutos por caixa $(52 \times 32 \times$ $17,5 \mathrm{~cm}$ )], frutos refugo (acima do tipo 12 , pequenos e deformados), massa fresca do fruto (kg/fruto) e número de frutos por planta. Foi obtida também a classificação dos frutos comerciais por tipo e o teor de sólidos solúveis totais ( ${ }^{\circ}$ brix), através de refratômetro de campo, a espessura de polpa e a relação de formato do fruto (razão comprimento/diâmetro do fruto), em amostras de quatro frutos representativos de cada parcela. A relação de formato do fruto (RF) classifi- cou os mesmos como esféricos $(\mathrm{RF}<1,0)$, oblongos $(1,1<\mathrm{RF}<1,7)$ e cilíndricos (RF>1,7) (Lopes, 1982). A significância dos efeitos dos fatores estudados sobre as características avaliadas foi determinada mediante a análise de variância e regressão polinomial, com base no modelo quadrático, ao nível de $5 \%$ de probabilidade, segundo metodologia descrita por Pimentel Gomes (2000). Os dados referentes à porcentagem foram transformados em arcoseno $\sqrt{P / 100}$

\section{RESULTADOS E DISCUSSÃO}

Houve efeitos significativos do espaçamento entre linhas e da densidade de plantas sobre as variáveis avaliadas, com exceção para a relação de formato de fruto e teor de sólidos solúveis totais que não apresentaram diferenças significativas.

A produtividade comercial diminuiu linearmente com a redução da densidade de plantio de 5,00 para 1,66 plantas por metro linear de sulco, ou seja, maiores produtividades foram alcançadas com as densidades de plantio mais elevadas. A densidade 5,00 plantas/metro linear proporcionada pelo espaçamento de 0,20 m entre plantas alcançou a maior produtividade com $40,69 \mathrm{t} / \mathrm{ha}$. A menor produtividade foi de $36,12 \mathrm{t} /$ ha obtida na densidade de 1,66 plantas por metro linear (0,60 m entre plantas) (Figura 1). Estes resultados corroboram aqueles obtidos por Grangeiro et al. (1999b), que verificaram maior produtividade com o 
Tabela 1. Equações de regressão para classificação percentual de frutos comerciais em tipos, de acordo com o tamanho e espessura de polpa do fruto (cm), em função de diferentes espaçamentos entre linhas e plantas. Juazeiro, Embrapa Semi-Árido, 1998.

\begin{tabular}{ll}
\hline \multicolumn{1}{c}{ Características } & \multicolumn{1}{c}{ Equações de regressão } \\
\hline & Classificação percentual de tipos de frutos \\
\hline Tipo 6 & $Y(1,80 \mathrm{~m})=3,6955+128,8118 E-120,0221^{* *} E^{2} R^{2}=1,00$ \\
& $Y(2,00 \mathrm{~m})=16,4305+45,4841^{* *} E \quad R 2=0,96$ \\
Tipo 8 & $Y(1,80 \mathrm{~m})=47,1068-31,3956 \mathrm{E}+44,9051^{* *} \mathrm{E}^{2} \quad R^{2}=1,00$ \\
& $Y(2,00 \mathrm{~m})=38,5831+30,0428 E-39,9539^{*} E^{2} \quad R^{2}=1,00$ \\
Tipo 10 & $Y(1,80 \mathrm{~m})=39,8225-30,7729^{* *} E \quad R^{2}=0,99$ \\
Tipo 12 & $Y(2,00 \mathrm{~m})=42,8215-44,1395^{* *} E \quad R^{2}=0,95$ \\
Espessura de polpa do fruto & $Y=38,1620-156,6214 E+156,6213^{* *} E^{2} \quad R^{2}=1,00$ \\
\hline
\end{tabular}

** Significativo ao nível de $1 \%$ de probabilidade, pelo teste de $\mathrm{F}$.

* Significativo ao nível de $5 \%$ de probabilidade, pelo teste de $\mathrm{F}$.

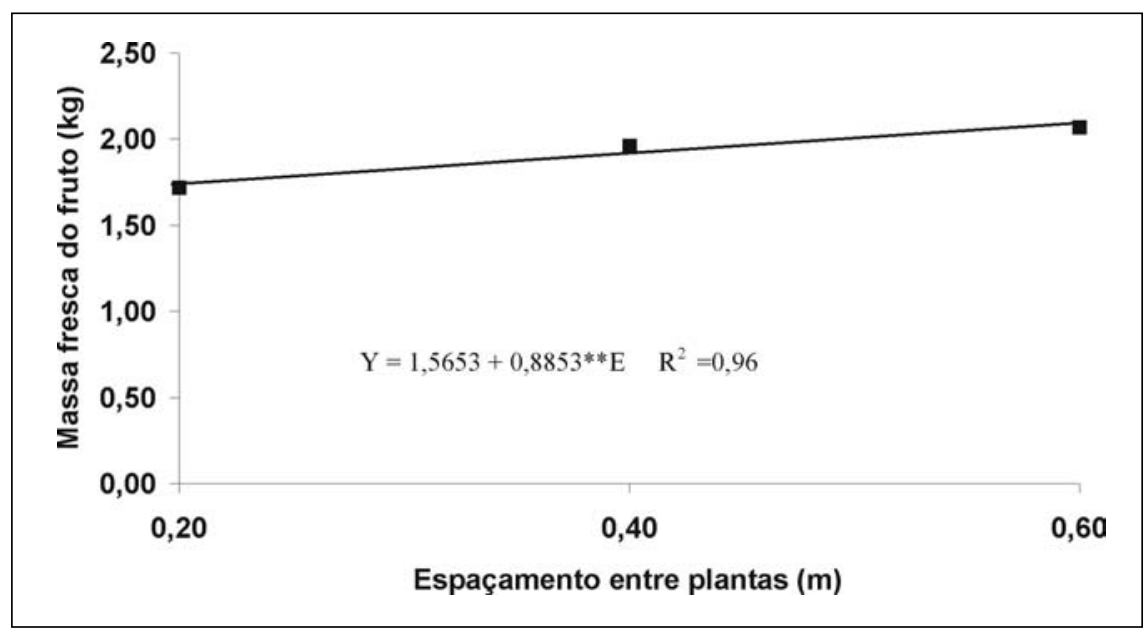

Figura 2. Massa fresca do fruto de melão em função do espaçamento entre plantas. Juazeiro(BA), Embrapa Semi-Árido, 1998.

aumento da densidade de plantas. Também Gualberto et al. (2001), constataram que a produtividade diminuiu significativamente, quando a densidade de plantio passou de 3,33 para 2,00 plantas por metro linear (espaçamento entre plantas de 0,30 para $0,50 \mathrm{~m}$ ), em cultivo de melão rendilhado, sob casa de vegetação.

Observou-se com a diminuição da densidade de plantio, uma menor produção de refugo, conforme se pode verificar pelo efeito linear negativo obtido para esta característica (Figura 1). Este fato deve-se ao maior tamanho de fruto proporcionado pelas densidades mais baixas. A densidade de 1,66 plantas metro linear proporcionada pelo espaçamento de $0,60 \mathrm{~m}$ entre plantas registrou a menor produção com 2,72 t/ ha (Tabela 1). Grangeiro (1997) e Paris et al. (1988), observaram maiores produções de frutos refugo com o aumento da densidade. Segundo os autores, isso pode ser atribuído à competição entre plantas por luz e nutrientes.

A massa fresca do fruto apresentou efeito linear positivo com a redução da densidade de plantio. A densidade de 1,66 plantas por metro linear apresentou a maior massa fresca do fruto com $2,09 \mathrm{~kg}$ /fruto (Figura 2). Pedrosa et al. (1991), relataram um aumento de $20 \%$ na massa fresca de frutos comerciáveis, na mesma densidade de plantio, comparada a 3,32 plantas por metro linear. Com relação à comercialização, verifica-se que no mercado interno, são preferidos os frutos maiores, com peso unitário de $2,0 \mathrm{~kg}$, tolerando-se uma variação de 1,0 a 2,0 kg (Filgueira, 2000). Já o mercado externo prefere frutos meno- res, com peso variando de 1,0 a $1,3 \mathrm{~kg}$ (Dusi, 1992), podendo chegar a 2,0 kg/ fruto (Gorgatti Neto et al., 1994).

Efeito linear positivo foi observado para número de frutos por planta, ou seja, com o aumento da densidade de plantio de 1,66 para 5,00 plantas por metro linear foi registrado um menor número de frutos. $\mathrm{O}$ espaçamento de $0,60 \mathrm{~m}$ com 1,66 plantas por metro linear sobressaiu-se com 2,10 frutos/planta, enquanto na maior densidade de plantas com 5,00 plantas por metro linear obteve-se 0,90 frutos/planta (Figura 3). Estes resultados estão de acordo com os obtidos por outros autores (Paris et al., 1988; Mendlinger, 1994, Grangeiro et al., 1999b; Kultur et al., 2001).

Assim, como as demais características avaliadas, a densidade de plantas influenciou a espessura de polpa do fruto (Tabela 1). À medida que se aumentou a densidade de plantio decresceu a espessura de polpa, sendo a maior espessura obtida com o espaçamento de 0,60 $\mathrm{m}$ entre plantas ou 1,66 plantas por metro linear. Observa-se que, da mesma forma como aconteceu para a massa fresca do fruto, também a ocorrência de frutos de maior espessura de polpa foi mais freqüente na menor densidade de plantas, demonstrando relação direta entre a massa fresca e espessura de polpa.

Não foram observados efeitos significativos sobre as características de relação de formato e teor de sólidos solúveis totais. Pedrosa et al. (1991) não verificaram efeito significativo da densidade de plantio em relação ao formato, enquanto Grangeiro et al. (1999a) 
observaram tendência dos frutos apresentarem formato arredondado, à medida que se aumentou a densidade de plantio. A relação de formato no melão é uma importante característica de qualidade, que interfere na embalagem do produto, transporte e comercialização. Frutos compridos, ocupam maior espaço e são de difícil acondicionamento. Assim como para relação de formato, os resultados para sólidos solúveis totais não evidenciaram diferenças significativas, variando entre 10,95 a $11,08 \%$. Estes resultados concordam com os obtidos por Grangeiro et al. (1999a) e Kultur et al. (2001), que relataram não haver diferenças para esta característica em relação à densidade de plantio. Segundo Dusi (1992) o teor de sólidos solúveis dos frutos (brix) está relacionado às condições climáticas de produção, onde a baixa umidade relativa do ar, aliada a altas temperaturas, proporcionam frutos com valores mais altos de brix, que são os de melhor aceitação de mercado.

Para frutos tipo 6 constatou-se efeito significativo da interação (Tabela 1). Verificou-se para o espaçamento de 1,80 $m$ entre linhas, um efeito quadrático com ponto de máxima porcentagem de frutos no espaçamento de $0,54 \mathrm{~m}$, o que equivale à densidade de 1,85 plantas por metro linear. Para o espaçamento de 2,00 $\mathrm{m}$ entre linhas evidenciou-se um efeito linear crescente com a redução da densidade de plantas.

Pela Tabela 1 observa-se efeito quadrático com ponto de mínima porcentagem de frutos para o espaçamento de $1,80 \mathrm{~m}$ entre linhas na densidade de 2,86 plantas por metro linear para a porcentagem de frutos tipo 8, sendo encontrados para o espaçamento de $2,00 \mathrm{~m}$, efeito quadrático com ponto de máxima porcentagem de frutos na densidade de 2,63 plantas por metro linear, proporcionado pelo espaçamento de 0,38 $\mathrm{m}$ entre plantas.

Com relação aos frutos de menor tamanho, verificou-se efeitos significativos da interação, com modelos lineares negativos para os frutos tipo 10 , ou seja, a porcentagem deste tipo de fruto reduziu-se com a diminuição da densidade de plantas nos dois espaçamentos entre linhas avaliados. Para os frutos tipo 12 , observou-se apenas efeito da den-

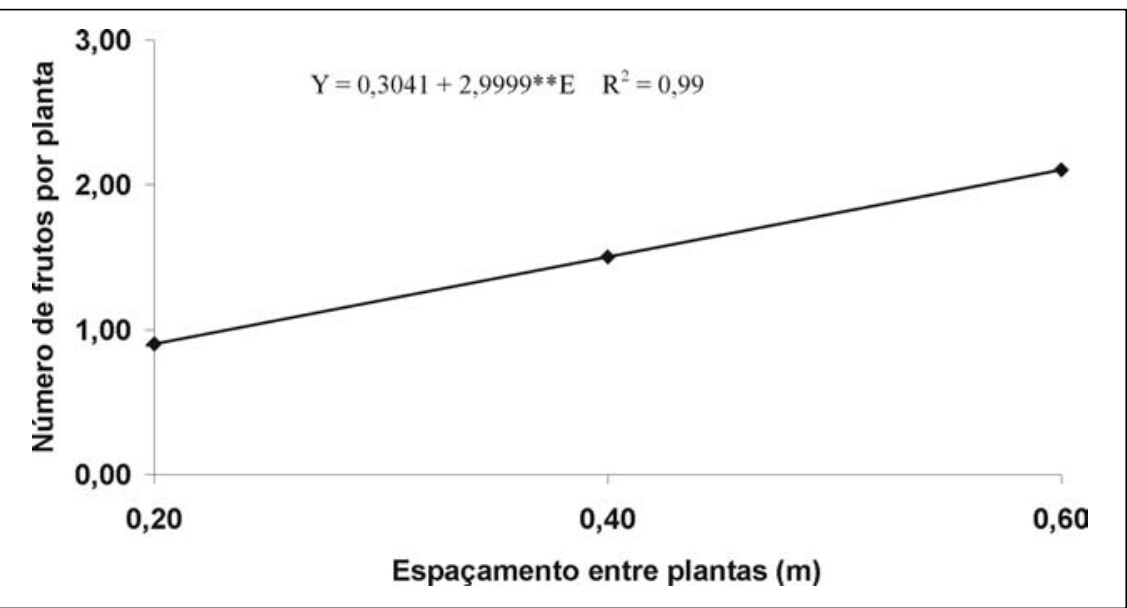

Figura 3. Número de frutos por planta do melão em função do espaçamento entre plantas. Juazeiro(BA), Embrapa Semi-Árido, 1998.

sidade de plantas, na qual a densidade de 2,00 plantas por metro linear representada pelo espaçamento de $0,50 \mathrm{~m}$ entre plantas, evidenciou a menor porcentagem de frutos nesta classificação (Tabela 1).

A maioria dos frutos concentrou-se nos tipos 6 e 8 , de maior tamanho e que têm a preferência do mercado interno. Já os frutos tipos 10 e 12, de melhor aceitação no mercado externo, foram menos freqüentes. Estes resultados são semelhantes aos obtidos por Grangeiro et al. (1999b) e Mendlinger (1994), que obtiveram uma maior percentagem de frutos de menor tamanho, com o incremento da densidade de plantio. Constatou-se com os resultados obtidos, a necessidade de se definir o mercado ao qual se destina o produto, para se estabelecer a melhor densidade de plantio.

Estes resultados evidenciam ser a densidade de plantio uma ferramenta de grande importância para os agricultores, podendo ser adequada em função do mercado consumidor para o qual se destina o produto. No entanto, pesquisas futuras são necessárias, procurando estudar outras densidades de plantio com maior número de plantas por cova, visando à máxima percentagem de frutos de menor tamanho dos tipos 10 e12, com o objetivo final de atender o mercado externo.

\section{LITERATURA CITADA}

ALMEIDA, J.H.S. Sistema de produção de melão cv. Valenciano Amarelo para o Estado do Rio Grande do Norte. Mossoró: ESAM, 1992. 43 p. Monografia. tura do melão. Lavras: Editora UFLA, 2002. 154

DIAS, R.C.S.; COSTA, N.D.; SILVA, P.C.G.; QUEROZ, M.A.; ZUZA, F.; LEITE, L.A.S.; PESSOA, P.F.A.P.; TARAO, D. A cadeia produtiva do melão no Nordeste. In. CASTRO, A.M.G.; LIMA, S.M.V.; GOEDART, W.J.; FREITAS, FILHO, A.; Vasconcelos, J.R.P., eds. Cadeia produtiva e sistemas naturais: prospecção tecnológica. Brasília: Embrapa - SPI, 1998. Cap. 17, p. 441- 494.

DUSI, A.N. Melão para exportação: aspectos técnicos da produção. Brasília DENACOOP, 1992. 38 p. (Série Publicações Técnicas, 1).

FAO (Roma, Italy). Agricultural production, primary crops. Disponível em <http:// www.fao.org >. Acesso em 13/11/02.

FARIA, C.M.B.; COSTA, N.D.; PINTO, J.M.; BRITO, L.T.L.; SOARES, J.M. Níveis de nitrogênio por fertirrigação e densidade de plantio na Agropecuária Brasileira, Brasília, v. 35, n. 3, p. 491-495, 2000.

FILGUEIRA, F.A.R. Novo Manual de olericultura: agrotecnologia moderna na produção e comercialização de hortaliças. Viçosa: UFV, 2000. 402 p.

GORGATTI NETO, A.; GAYET, J.P.; BLEINROTH, E.W.; MATALLO, M.; GARCIA E.E.C.; GARCIA, A.E.; ARDITO, E.F.G.; BORDIN, M.R. Melão para exportação: procedimentos de colheita e de pós-colheita. Brasília: EMBRAPA - SPI/FRUPEX, 1994, 37 p. (EMBRAPA - SPI. Publicações Técnicas, 6).

GRANGEIRO, L.C. Densidade de plantio em hibridos de melão amarelo. Mossoró: ESAM, 1997. 48 p. (Tese mestrado).

GRANGEIRO, L.C.; PEDROSA, J.F.; BEZERRA NETO, F.; NEGREIROS, M.Z. Qualidade de híbridos de melão em diferentes densidades de plantio. Horticultura Brasileira, Brasília, v. 17, n. 2, p. 110-113, 1999a.

GRANGEIRO, L.C.; PEDROSA, J.F.; BEZERRA NETO, F.; NEGREIROS, M.Z. Rendimento de híbridos de melão em diferentes densidades de plantio. Horticultura Brasileira, Brasília, v. 17, n. 3, p. $200-206,1999$ b.
ALVARENGA, M.A.R.; RESENDE, G.M. Culp. (UFLA, Textos Acadêmicos, 20). cultura do melão em um vertissolo. Pesquisa 
GUALBERTO, R.; RESENDE, F.V.; LOSASSO,

P.H.L. Produtividade e qualidade do melão rendilhado em ambiente protegido, em função do espaçamento e sistema de condução. Horticultura Brasileira, Brasília, v. 19, n. 3, p. 373-376, 2001. IBGE. Indicadores conjunturais - produção agricola/agricultura. Disponível em <http:// www.Ibge.gov.br>, Acesso em 13/11/02.

KULTUR, F.; HARRISON, H.C.; STAUB, J.E. Spacing and genotype affect fruit sugar concentration, yield, and fruit size of muskmelon. HortScience, v. 36, n. 2, p. 274-278, 2001.

LOPES, J.F. Melhoramento genético (chuchu, melancia, melão e pepino). Informe Agropecuário, Belo Horizonte, v. 8, n. 85, p. 61-65, 1982.
MAKISHIMA, N. Situação das cucurbitáceas no Brasil. Horticultura Brasileira, Brasília, v. 9, n. 2, p. 99-101, 1991.

MENDLINGER, S. Effect of increasing plant density and salinity on yield and fruit quality in muskmelon. Scientia Horticulturae, v. 57, n. 1-2, p. 41-49, 1994.

PARIS, H.S.; NERSON, H.; BURGER, Y.; EDELSTEIN, M.; KARCHI, Z. Synchrony of yield of melons affected by plants type and density. Journal Horticultural Science, v. 63, n. 1, p. 141147, 1988.
PEDROSA, J.F. Cultura do melão. Petrolina - PE, 1994. 25 p. (Mimeografado )

PEDROSA, J.F.; TORRES FILHO, J.; MEDEIROS, I.B. Poda e densidade de plantio em melão. Horticultura Brasileira, Brasília, v. 9, n. 1, p. 18-22, 1991.

PIMENTEL GOMES, F. Curso de estatística experimental. 14. ed. São Paulo: Nobel, 2000. 477 p.

ROBINSON, R.W.; WALTERS, D.S.D. Cucurbits. New York: CAB International, 1997. 226 p. 